\title{
Linfohistiocitosis hemofagocítica en trasplante renal
}

\author{
John Fredy Nieto-Ríos ${ }^{1,2}$, Carol Lisbeth Morales-Contreras ${ }^{3}$, Diana Carolina Chacón-Jaimes ${ }^{3}$, Diego Armando Benavides- \\ Henao ${ }^{3}$, Diana Carolina Bello-Márquez ${ }^{4}$, Lina María Serna-Higuita ${ }^{5}$
}

\section{RESUMEN}

La linfohistiocitosis hemofagocítica (LHH) posterior al trasplante renal hace referencia a un estado hiperinflamatorio grave, asociado a la activación no controlada de los linfocitos T citotóxicos y macrófagos por causa infecciosas y/o secundaria al tratamiento inmunosupresor. Las causas más prevalentes dentro de las infecciones son la histoplasmosis, la tuberculosis y las infecciones por virus herpes. Se caracteriza por fiebre, organomegalias, citopenias, hiperferritinemia, hipertrigliceridemia y/o hipofibrinogenemia; puede acompañarse con hemofagocitosis documentada en la médula ósea, el hígado u otros órganos. Su curso puede ser fulminante con progresión a falla multisistémica y la muerte.

El tratamiento va enfocado a controlar tempranamente la causa desencadenante, reducir la inmunosupresión y controlar la inflamación. En pocos casos es necesario el uso de otros inmunosupresores, quimioterapia o, en situaciones muy seleccionadas, se puede requerir el trasplante de médula ósea.

\section{PALABRAS CLAVE}

Ferritinas; Histoplasmosis; Linfohistiocitosis Hemofagocítica; Trasplante RenaI

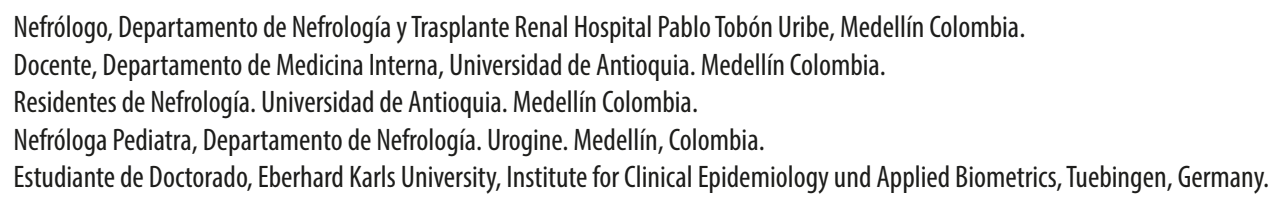

Cómo citar: Nieto-Ríos JF, Morales-Contreras CL, Chacón-Jaimes DC, Benavides-Henao DA, Bello-Márquez DC, Serna-Higuita LM. Linfohistiocitosis hemofagocítica en trasplante renal. latreia. 2019 0ct-Dic;32(4):311-320. DOI 10.17533/udea.iatreia.21. 


\section{SUMMARY}

\section{Haemophagocytic lymphohistiocytosis in kidney transplant recipients}

Hemophagocytic lymphohistiocytosis (HLH) in renal transplant recipients is a life-threatening hyper-inflammatory syndrome; associated with uncontrolled activation of cytotoxic T-lymphocytes and macrophages due to infections or immunosuppressive therapy. Histoplasmosis, tuberculosis and herpes virus infection are among the leading infectious causes. It is characterized by fever, oroganomegaly, cytopenia, hyperferritinemia, hypertrigiceridemia and/or hypofibrinogenemia; which may be accompanied by hemophagocytosis in bone marrow, liver or other organs. HLH can follow a rapidly fatal course, with progression to multisystemic failure and death. The treatment is based on early control of the triggering cause, reducing immunosuppression and stop the inflammatory process. In some cases, is necessary to use other immunosuppressant, chemotherapy and in a very few cases, a bone marrow transplant may be required.

\section{KEY WORDS}

Hemophagocytic Lymphohistiocytosis; Histoplasmosis; Ferritins; Kidney Transplantation

\section{INTRODUCCIÓN}

La linfohistiocitosis hemofagocítica (LHH), más conocida en la literatura médica como síndrome hemofagocítico, es caracterizada por una activación excesiva de naturaleza no maligna de los macrófagos y los linfocitos T citotóxicos (LTc), como resultado de una citotoxicidad defectuosa asociada a una marcada hipercitoquinemia ${ }^{(1)}$. Fue descrita por primera vez en 1939, pero su caracterización se logró en 1979 y desde entonces se han realizado múltiples modificaciones en sus criterios diagnósticos ${ }^{(2)}$.

La LHH se clasifica en primaria y secundaria, de acuerdo con la presencia o no de defectos genéticos, respectivamente (Tabla 1). La LHH primaria a su vez se subdivide en linfohistiocitosis hemofagocítica familiar con herencia autosómica recesiva, causada por mutaciones en los genes implicados en la expresión de las proteínas de tráfico de los gránulos citotóxicos ${ }^{(3)}$; LHH asociada a síndromes genéticos (Chediak Higashi, Griselli y Hermansky Pudlak) y la enfermedad linfoproliferativa asociada al cromosoma $\mathrm{X}$. Usualmente las manifestaciones clínicas de las LHH primarias se presentan en los primeros 12 meses de vida, con una supervivencia promedio aproximada de 10 años ${ }^{(2,4,5)}$. Las formas secundarias o adquiridas se manifiestan principalmente en la población adulta, pero pueden manifestarse en la población infantil ${ }^{(6-13)}$, presentándose frecuentemente en el contexto de las infecciones, tumores malignos y enfermedades autoinmunes.

Adicionalmente, se han reportado algunos casos de LHH en pacientes con trasplante de órgano sólido bajo terapia inmunosupresora, con una incidencia reportada de $0,4-0,5 \%{ }^{(14)} y$ con una mortalidad del $80 \%$, especialmente cuando se asocia con una falla multisistémi$\mathrm{ca}^{(2,4,15,16)}$. Karras y col. observaron que la presencia de organomegalia, elevación de las aminotransferasas, prolongación del tiempo de protrombina y trombocitopenia, fueron factores de riesgo de muerte en pacientes con LHH posterior a un trasplante renaI ${ }^{\left({ }^{15}\right)}$.

\section{Tabla 1. Clasificación de la linfohistiocitosis hemofagocítica}

\begin{tabular}{cc}
\hline LHH PRIMARIA & LHH SECUNDARIA \\
Linfohistiocitosis hemofagocítica & \\
familiar tipo 1 al 5 & Infecciones \\
Asociada a síndromes genéticos: & Cáncer \\
síndrome de Chediak Higashi & Medicamentos \\
síndrome de Grisselli & Desórdenes autoinmu- \\
síndrome de Hermansky Pudlak & nes, autoinflamatorios \\
Enfermedad linfoproliferativa & \\
asociado al cromosoma X & \\
\hline Fuente: ${ }^{(3)}$ &
\end{tabular}

En la actualidad existe poca literatura con respecto a la LHH en pacientes trasplantados de riñón, siendo en su mayoría proveniente de reportes o series de casos (Tabla 2) ${ }^{(11,12,14,17-20)}$. El poco conocimiento de la LHH postrasplante, sumado a su presentación clínica inespecífica, hace que su diagnóstico sea un reto. Esta revisión pretende evaluar el estado de arte actual de la LHH postrasplante renal, permitiendo al clínico adquirir un conocimiento actualizado sobre la fisiopatología, evolución clínica, el diagnóstico y tratamiento de esta entidad. 
Tabla 2. Reporte de casos de LHH en trasplante renal

\begin{tabular}{|c|c|c|c|c|c|c|c|}
\hline \multicolumn{8}{|c|}{ Series de casos } \\
\hline Año (ref) & n.o pacientes & $\begin{array}{c}\text { Causa } \\
\text { n. }{ }^{\circ}(\%)^{*}\end{array}$ & $\begin{array}{c}\text { Reducción } \\
\text { Inmunosupresión }\end{array}$ & Rechazo & Qx & Muerte & $\begin{array}{l}\text { Pérdida del } \\
\text { injerto }\end{array}$ \\
\hline $1979(21)$ & 19 & $14(73,7 \%)$ & $14(73,7 \%)$ & Sí & No & No & Sí \\
\hline 2004 (15) & 17 & $13(76,5 \%)$ & $16(94,1 \%)$ & Sí & No & No & Sí \\
\hline \multicolumn{8}{|c|}{ Reportes de casos } \\
\hline Año (ref) & Días postrasplante & Condición asociada & $\begin{array}{l}\text { Inducción depletores } \\
\text { linfocitos }\end{array}$ & $\begin{array}{c}\text { Reducción } \\
\text { inmunosupresión }\end{array}$ & Qx & Muerte & $\begin{array}{l}\text { Pérdida del } \\
\text { injerto }\end{array}$ \\
\hline $2016(11)$ & 5 & CMV & Sí & No & No & Sí & - \\
\hline $2013(14)$ & 36 & Rechazo agudo & Sí & Sí & No & No & No \\
\hline $2014(12)$ & 300 & BK & Sí & Sí & No & No & Sí \\
\hline 2006 (18) & 10 & Toxoplasm & Sí & Sí & No & Sí & - \\
\hline 2008 (19) & 700 & PVB19 & No descrito & Sí & No & No & No \\
\hline
\end{tabular}

Qx: quimioterapia. *: causa identificada que desencadenó la LHH. CMV: infección por citomegalovirus. BK: nefropatía por BK virus. Toxoplasm: toxoplasmosis. PVB19: parvovirus B19. Fuente: $(11,12,14,15,18,19,21)$

\section{FACTORES DE RIESGO}

Las formas secundarias de la LHH están relacionadas con una respuesta inmune alterada, lo cual es una característica de los pacientes inmunosuprimidos, bien sea por infecciones o por tratamiento para enfermedades autoinmunes, neoplásicas o trasplante (Tabla 1) ${ }^{(6-12)}$. En los pacientes trasplantados esta condición se puede presentar especialmente en las primeras semanas postrasplante $y$ se relaciona frecuentemente con infecciones, la presencia de malionidades y el uso de medicamentos depletores de linfocitos ${ }^{(22)}$.

Existe una relación compleja entre los procesos infecciosos y la LHH, esto debido a las infecciones que pueden causar este síndrome en personas predispuestas genéticamente o a la leucopenia secundaria a la
LHH que puede favorecer el desarrollo de un proceso infeccioso. Los microorganismos más frecuentemente asociados son los virus, especialmente los herpesvirus, por infección primaria o reactivación. También se han descrito infecciones bacterianas, micobacterianas, parasitarias y fúngicas (Tabla 3) ${ }^{(2)}$. En relación a las infecciones fúngicas, los hongos del género histoplasma son los causantes más frecuentes de LHH en los pacientes inmunosuprimidos por el virus de la inmunodeficiencia humana (VIH) y por el trasplante renal ${ }^{(8-10)}$; hallazgo a tener en cuenta ya que la histoplasmosis a su vez se presenta en forma no despreciable en los pacientes trasplantados de órogano sólido, un estudio realizado en la ciudad de Medellín reportó una incidencia del 1,1 \% en los pacientes trasplantados renales ${ }^{(9)}$.

Tabla 3. Causas infecciosas del síndrome hemofagocítico

\begin{tabular}{|c|c|c|}
\hline Virus & Bacterias & Parásitos y hongos \\
\hline 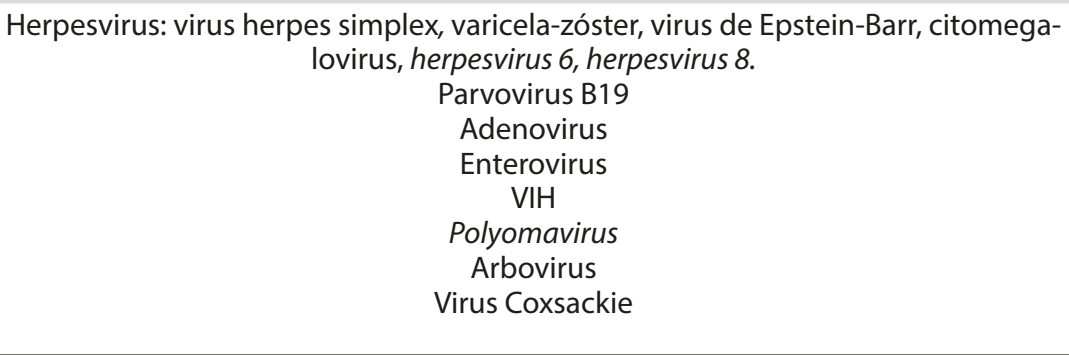 & $\begin{array}{c}\text { Micobacterias } \\
\text { Salmonella } \\
\text { Borrelia burgdorferi } \\
\text { Leptospira } \\
\text { Brucella tularensis } \\
\text { Chlamydia psittaci } \\
\text { Mycoplasma pneumoniae } \\
\text { Ehrlichia } \\
\text { Rickettsias } \\
\text { Legionella }\end{array}$ & $\begin{array}{l}\text { Babesia } \\
\text { Leishmania } \\
\text { Toxoplasma gondii } \\
\text { Plasmodium } \\
\text { Pneumocystis jirovecii } \\
\text { Candida } \\
\text { Aspergillus } \\
\text { Cryptococcus } \\
\text { Histoplasma }\end{array}$ \\
\hline
\end{tabular}

Fuente: (2) 
De forma similar, existe una relación circular entre los procesos malignos y la LHH. Esta asociación es mayor con los tumores malignos linfoproliferativos (Linfoma de células $T$ \& B), específicamente en los inducidos por virus, principalmente el virus Epstein-Barr (EBV), siendo uno de los más frecuentes en la enfermedad linfoproliferativa asociada al trasplante (PTLD, del inglés Posttransplant lymphoproliferative disease) ${ }^{(23-25)}$. Con menos frecuencia se asocia a tumores malignos sólidos ${ }^{(26,27)}$, los cuales también se pueden presentar luego del trasplante. En las enfermedades autoinmunes (artritis crónica juvenil, enfermedad de Still, lupus eritematoso sistémico, esclerosis sistémica, artritis reumatoide), la LHH se puede presentar espontáneamente o relacionada con la terapia inmunosupresora y las infecciones. Por otro lado, en los últimos años han incrementado los reportes de casos que asocian esta entidad con la administración de biológicos (nivolumap, ipilimumab, etanercept, prembrolizumab) (28), fármacos convencionales (vancomicina, trimetropim-sulfametoxazol, fenobarbital, lamotrigina, fenitoina, metrotexate, sulfasalazina, ácido retinoico) y nutrición parenteral, terapias que son utilizadas frecuentemente en los pacientes trasplantados ${ }^{(6,29)}$.

\section{FISIOPATOLOGÍA}

En condiciones normales ante una infección viral o en la presencia de células tumorales, las células que presentan antígenos activan los linfocitos CD8+ citotóxicos (LTc) y las células Natural Killer (NK), las cuales eliminan las células infectadas (o células tumorales) por medio de la lisis mediada por perforinas (Figura 1a). Los LTc responden a la activación de sus receptores por patrones moleculares asociados a daño (DAMPS) o a patógenos (PAMPS). Posterior a su activación, estas células producen a nivel intracelular granzimas (Gz) y perforinas (Perf) por el aparato de Golgi. Una vez formado el gránulo citotóxico, este migra a la membrana celular por la señalización ofrecida por las proteínas en la pared del gránulo, entre ellas las proteínas munc 13-4, munc 18-2 y la sintaxina 11 que favorecen su adhesión y posterior liberación en la sinapsis inmunológica (Figura 1b) ${ }^{(30)}$.

Aunque la forma de acción reconocida para la citotoxidad corresponde a la formación de poros en las células diana (células presentadoras de antígenos)
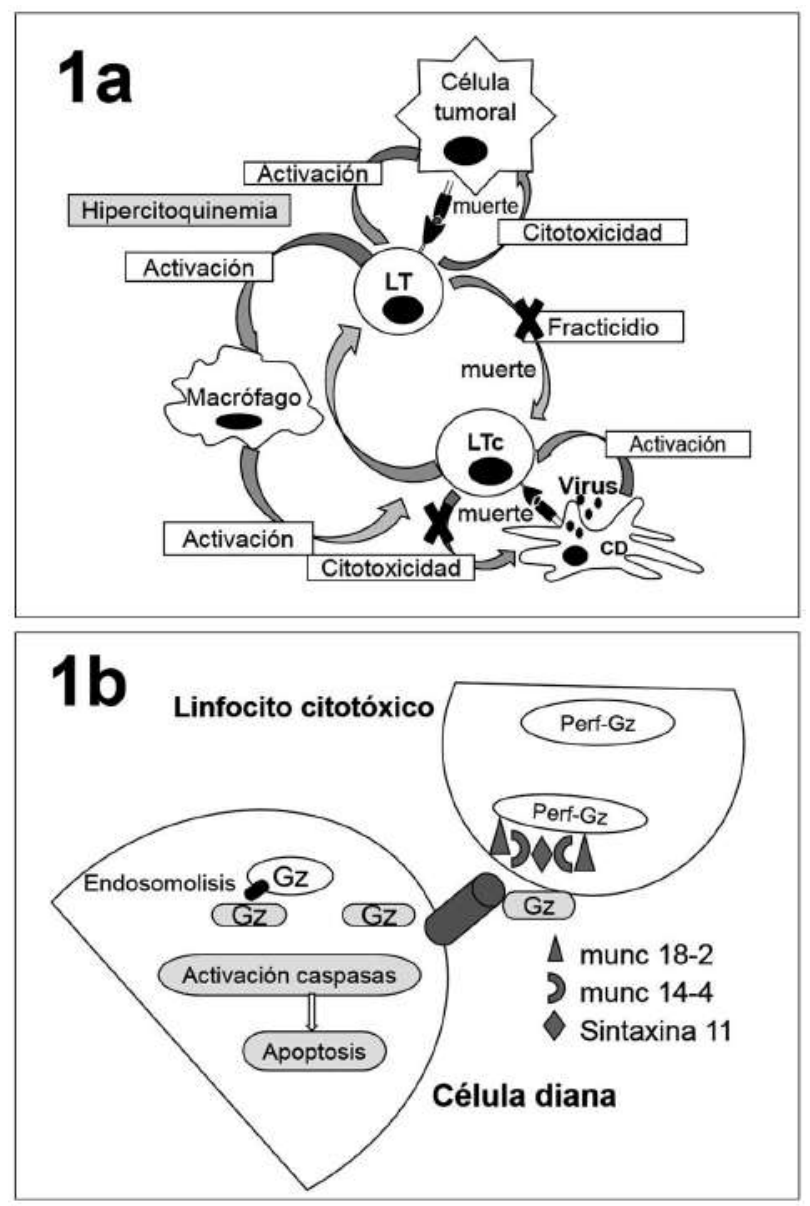

\section{Figura 1. Fisiopatología de la LHH}

En la figura 1a se muestra la activación de los linfocitos T citotóxicos (LTC) por las células dendríticas, la cual lleva a la lisis celular mediada por perforinas. Estos LTc a su vez se eliminan ellos mismos mediante lisis celular mediada por perforinas (fractricidio). En la LHH postrasplante se postula que el uso de medicamentos inmunosupresores induce un deterioro del fracticidio y la eliminación de las células presentadoras de antígenos (vías señaladas con " $X$ "), lo cual perpetúa la respuesta inmune. Figura $1 \mathrm{~b}$ : esquema de la citotoxicidad mediada por gránulos, en esta se observan las proteínas presentes en los gránulos citotóxicos de los LTc y la liberación de estos hacia las células diana. LTc: linfocito. C citotóxico. CD: célula presentadora de antígenos. Gz: granzimas. Perf: perforinas. Fuente: Modificado de Morimoto et al. y Voskoboinik et al. ${ }^{(4,30)}$.

por las Perf y posterior penetración de las Gz; en años recientes se han identificado procesos de endocitosis de las Gz y Perf, con el posterior anclaje de las Perf en la pared de los endosomas y la liberación 
intracelular de las Gz. Estas últimas interactúan y clivan los dominios de los agonistas de muerte (BID: BH3 interacting-domain death agonist), que a su vez permiten la permeabilización de la membrana externa mitocondrial para la activación de citocromos y caspasas, que inducen apoptosis celular (Fioura 1b).

La activación de las NK y LTc favorece, además, la liberación de citoquinas proinflamatorias (interferón $\gamma$, factor de necrosis tumoral $\alpha$, interleucinas IL-1b, IL-2, IL-6, IL-12, IL-18, entre otras) que estimulan a otras células, entre ellos los macrófagos, los cuales incrementan la activación de los LTc. Esta repuesta citotóxica está limitada mediante diferentes mecanismos, siendo el fratricidio (muerte entre LTc mediante lisis celular mediada por perforinas) uno de los mayores reguladores. Diferentes estudios han identificado como causantes de las LHH hereditarias (primarias) las mutaciones en genes relacionados con la síntesis o secreción de los gránulos citotóxicos de los LTc y las células NK. Por el contrario, en las LHH secundarias no es clara la causa que desencadena este proceso. Se postula que el uso de medicamentos inmunosupresores induce un deterioro de la actividad citotóxica de las células NK y los LTc para eliminar los procesos infecciosos o tumorales y un bloqueo del fratricidio ${ }^{(31)}$; lo que lleva a una expansión no controlada del LTc con la consecuente perpetuación de la respuesta inmune y la secreción continua de citoquinas, activando macrófagos que a su vez producen más citoquinas para terminar, finalmente, en una tormenta de citoquinas con un grave síndrome de respuesta inflamatoria y falla orgánica multisistémica (Figura 1a) ${ }^{(17,30-37)}$.

\section{MANIFESTACIONES CLÍNICAS}

Por lo general, el cuadro clínico se instaura de forma aguda ocurriendo en las primeras semanas luego del trasplante renal, cuando el paciente está intensamente inmunosuprimido, o también luego de recibir el tratamiento para el rechazo agudo ${ }^{(14)}$. En algunos pacientes la LHH ha sido reportada años después del trasplante, asociada a la presencia de neoplasias, parasitosis, infección por parvovirus B19 e histoplasmosis ${ }^{(17)}$. Los síntomas y signos más representativos son el marcado compromiso del estado general: fiebre alta, pérdida de peso, anorexia, linfadenopatías generalizadas y hepatoesplenomegalia por infiltración de macrófagos ${ }^{(15,21,38)}$. Con menos frecuencia se encuentra rash cutáneo, púrpura, edemas, compromiso del sistema nervioso central que puede ser diverso, manifestándose como confusión, cefalea, meningitis, convulsiones y encefalopatía ${ }^{(1,4,29)}$. En una serie de 17 pacientes con trasplante renal y diagnóstico de LHH, 7 de ellos presentaron síntomas neurológicos, siendo el estupor y las convulsiones los más frecuentes ${ }^{(15)}$. También puede presentarse un compromiso del injerto renal con un cuadro histológico muy variado, como se describe a continuación.

Lesiones tubulointersticiales: son las más frecuentes y se caracterizan por el desarrollo de necrosis tubular aguda, con marcado infiltrado inflamatorio y la presencia de hemofagocitos. Entre el 30-50\% de los pacientes desarrollan una lesión renal aguda que se agrava con el uso de medicamentos nefrotóxicos y la falla multisistémica. Los estudios concluyen que la hipercitoquinemia, especialmente el incremento en los niveles del factor de necrosis tumoral $\alpha$ (FNT- $\alpha$ ), produce apoptosis tubular a través de las vías de señalización ASK1 (apoptosis sionnal-regulating kinase 1) ${ }^{(2,39)}$.

Lesiones glomerulares: entre los hallazgos histológicos reportados se encuentran la glomerulopatía variedad colapsante y la enfermedad de cambios mínimos. Adicionalmente, se observa una pérdida de la arquitectura de los podocitos sin acúmulos de complejos inmunes. También el FNT- $\alpha$ está aumentado, favoreciendo indirectamente los factores de transcripción y reorganización del citoesqueleto de actina ${ }^{(40,41)}$.

Lesiones vasculares: el prototipo es la microangiopatía trombótica secundaria a una lesión endotelial por la tormenta de citoquinas ${ }^{(2,39)}$.

\section{ESTUDIOS DIAGNÓSTICOS}

El diagnóstico de la LHH es un reto, ya que los hallazosos clínicos y de laboratorio pueden ser similares a los observados en otras enfermedades como infecciones, rechazo, cáncer o los efectos secundarios de los inmunosupresores u otros medicamentos. Todo esto obstaculiza el diagnóstico precoz de esta entidad (17). Con respecto a los exámenes de laboratorio, la hiperferritinemia es considerada uno de los hallazogos más útiles para el diagnóstico de LHH, especialmente cuando supera $10.000 \mathrm{ug} / \mathrm{mI}$, con una sensibilidad y especificidad de 90 y $96 \%$, respectivamente ${ }^{(2,42,43)}$. En el hemograma se puede encontrar un compromiso de dos o tres de las líneas celulares. Se presenta además 
hipertrigliceridemia, la cual es secundaria a la inhibición de la lipoproteinlipasa endotelial por el FNT- $\alpha$. Dicha enzima que tiene como función la hidrólisis de triglicéridos para formar quilomicrones y VLDL.

Por lesión tisular puede haber un incremento de la deshidrogenasa láctica (LDH) y la creatinkinasa (CPK). Las pérdidas de líquidos por lesiones capilares inducen a hipoalbuminemia e hiponatremias de grado variable $^{(1,4,5,29) \text {. }}$

En laboratorios clínicos de referencia se pueden analizar los niveles del receptor soluble de la interleucina 2 (sIL2-R) o CD25, el cual es un marcador de la activación de linfocitos. Para su diagnóstico se precisan valores mayores a $2.400 \mathrm{UI} / \mathrm{mI}$. La citometría de flujo se puede utilizar para evaluar la actividad de las NK, los niveles de perforinas en los LTc y el marcador de membrana de células citotóxicas CD107a, que hace parte de los gránulos que liberan Perf y Gz ${ }^{(1,29,44,45)}$. En la biopsia de médula ósea se puede observar la presencia de eritrofagocitosis medular, hallazgo característico de la LHH (14); sin embarogo, su ausencia no excluye el diagnóstico. En caso de una alta sospecha, se recomienda la toma de biopsias de médula ósea seriadas (Figura 2)(45). Adicionalmente, esta hemofagocitosis se puede observar en el tejido esplénico, hepático, renal, los ganglios linfáticos y la piel ${ }^{(14,44)}$.

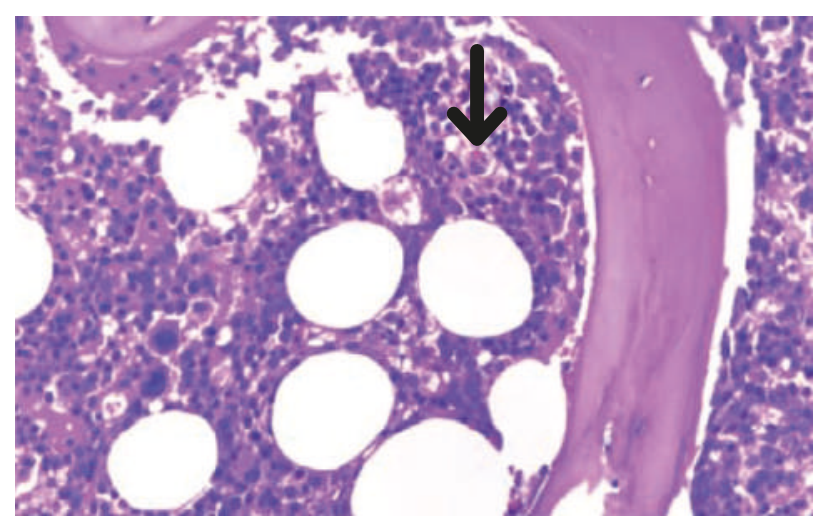

\section{Figura 2. Hallazgos histológicos en la médula ósea}

Biopsia de médula ósea (Giemsa, 40X), se observa la médula ósea hipocelular con la presencia de hemofagocito (flecha negra).

Fuente: consentimiento, Nieto-Ríos et al. ${ }^{(8)}$
Ante la sospecha de compromiso neurológico se recomienda realizar, -a menos que la condición clínica la contraindique-, una punción lumbar para evaluar en el líquido cefalorraquídeo la presencia de pleocitosis (células mononucleares), proteinorraquia y, raramente, hemofagocitos ${ }^{(1)}$. Otros de los hallazgos de los laboratorios reportados en pacientes con LHH postrasplante renal son las transaminasas elevadas, la hiperbilirrubinemia y alteración leve de las pruebas de coagulación ${ }^{(15)}$.

La sociedad internacional del histiocito, en el 2004 , actualizó los criterios clínicos y de laboratorio para el diagnóstico de LHH, el cual se hace con la confirmación molecular de las alteraciones genéticas o la presencia de 5 de los 8 criterios enlistados en la Tabla $4^{(4,46)}$.

\section{Tabla 4. Criterios diagnósticos para LHH (2004)}

\begin{tabular}{l} 
A. Diagnóstico molecular consistente con LHH \\
(PRF1, UNC13D, STXBP2, RAB27A, STX11, SH2D1A o XIAP) \\
B.Presencia de cinco de los ocho criterios clínicos siguientes: \\
1. Fiebre \\
2. Esplenomegalia \\
3. Bicitopenia \\
4. Hipertrigliceridemia ( $>265 \mathrm{mg} / \mathrm{dl}$ ) o hipofibrinogenemia \\
$(<1,5 \mathrm{~g} / \mathrm{l})$ \\
5. Hemofagocitosis \\
6. Actividad células NK, baja o ausente \\
7. Hiperferritinemia (> $500 \mu \mathrm{mg} / \mathrm{I})$ \\
8. Aumento de los niveles de CD25 solubles (receptor IL-2) \\
en suero ( $>2,400 \mathrm{UI} / \mathrm{ml})$ \\
\hline
\end{tabular}

Fuente: ${ }^{(4,46)}$

Posterior al diagnóstico, es fundamental encontrar la causa que desencadenó la LHH ${ }^{(17)}$. La primera causa para considerar en la población trasplantada son las infecciones (virales, bacterianas, micobacterianas, micóticas, parasitarias). Para esto, se deben realizar los estudios microbiológicos respectivos que incluyen policultivos (sangre, médula ósea, tracto respiratorio, urinario, gastrointestinal), pruebas moleculares (cargas virales) y biopsias, entre otros. En la población colombiana con trasplante de riñón la causa más frecuente de LHH es la histoplasmosis diseminada ${ }^{(8-10)}$. Otras posibles causas que deben ser evaluadas son la presencia 
de tumores malignos, en especial la enfermedad linfoproliferativa asociada al trasplante ${ }^{(24,25)}$. Para dicha evaluación se deben realizar estudios imaginológicos corporales totales (TAC, resonancias, PET-CT), estudios moleculares deI EBV, tanto en la sangre como en el tejido, estudios complementarios de médula ósea y biopsias de ganglios linfáticos u órganos comprometidos ${ }^{(24,}$ ${ }^{25)}$. De no encontrarse la causa, se deben considerar las enfermedades autoinmunes, la enfermedad del injerto contra huésped y, por último, las causas tóxicas o medicamentosas. En la población infantil se debe tener en cuenta desde el principio las causas genéticas ${ }^{(24)}$.

\section{TRATAMIENTO}

La meta del tratamiento de Ia LHH es establecer un diagnóstico temprano antes de que se instaure la falla orgánica multisistémica. Los objetivos están encaminados a controlar la enfermedad de base (infección, neoplasia maligna, autoinmunidad, toxicidad, genética), atenuar a los macrófagos activados y limitar el estado de hiperinflamación ${ }^{(17)}$. De no encontrarse prontamente la causa de la LHH y de no instaurarse un tratamiento oportuno y eficaz para esta causa encontrada, el resultado final será la falla multisistémica y la muerte del paciente ${ }^{(10)}$.

El tratamiento coadyurante de Ia LHH en los pacientes trasplantados de riñón se basa en la reducción de Ia dosis de inmunosupresores, vigilando la presencia de episodios de rechazo y el adecuado control de las infecciones con el manejo antimicrobiano apropiađo. Se recomienda además, aunque sin mucha evidencia, el uso de esteroides a altas dosis con tiempos de duración no claramente establecidos ${ }^{(2,39)}$. Existen escasos reportes del uso de la inmunoglobulina polivalente a dosis de $0,5 \mathrm{~g} / \mathrm{k}$ una vez por semana, lo que podría ayudar en el manejo ${ }^{(1,47)}$.

La quimioterapia con etopósido está reservada para casos muy esporádicos que son resistentes al manejo y para las formas genéticas ${ }^{(4,6,48)}$. Estos pacientes podrían beneficiarse del trasplante de médula ósea, con posibilidad de supervivencia a los 3 años del $57 \%$, en los casos familiares ${ }^{(48)}$. Algunos grupos han implementado terapias de rescate con plasmaféresis para la eliminación de citoquinas $(4,17,49,50)$, biológicos inhibidores del FNT- $\alpha$ (etanercep, infliximab, adalimubab, golimubab, certolizumab) y alemtuzumab, anticuerpo monoclonal humanizado contra la glicoproteína de superficie celular CD52 con resultados variables ${ }^{(51)}$.

Infortunadamente, el tratamiento de la LHH aún no está definido y los estudios donde se han evaluado pacientes trasplantados renales con LHH son muy heterogéneos, con un bajo número de pacientes y diferentes combinaciones de medicamentos; por lo que la decisión terapéutica, en muchas ocasiones, se basa en la experiencia más que en los datos disponibles. Se sugiere en estos pacientes la búsqueda de las causas que llevaron a esta enfermedad, sumado a la terapia coadyuvante, todo esto encaminado a reducir la mortalidad de la LHH.

\section{CONCLUSIÓN}

La LHH es una complicación rara que puede presentarse posteriormente al trasplante renal, esta se relaciona generalmente, con causas secundarias como los medicamentos inmunosupresores, episodios de rechazo, las infecciones, neoplasias, tóxicos y enfermedades autoinmunes, entre otros. El curso clínico generalmente es fulminante $y$ se produce alta morbimortalidad; por lo cual esta entidad se debe sospechar tempranamente, cuando los pacientes presentan fiebre, organomegalias y/o citopenias. En los estudios complementarios usualmente se encuentra hiperferritinemia, hipertrigliceridemia, hipofibrinogenemia, hemofagocitosis, elevación del CD25 soluble y una alteración en la actiøidad de las células NK. En la actualidad no existe un tratamiento definitivo, por este motivo hacemos énfasis en la necesidad de un diagnóstico precoz y el control oportuno de la causa desencadenante.

\section{CONFLICTOS DE INTERESES}

Ninguno por declarar.

\section{REFERENCIAS BIBLIOGRÁFICAS}

1. Ramachandran S, Zaidi F, Aggarwal A, Gera R. Recent advances in diagnostic and therapeutic guidelines for primary and secondary hemophagocytic 
lymphohistiocytosis. Blood Cells Mol Dis. 2017;64:537. DOI 10.1016/j.bcmd.2016.10.023.

2. Karras A. What nephrologists need to know about hemophagocytic syndrome. Nat Rev Nephrol. 2009;5(6):329-36. DOI 10.1038/nrneph.2009.73.

3. Otrock ZK, Eby CS. Clinical characteristics, prognostic factors, and outcomes of adult patients with hemophagocytic lymphohistiocytosis. Am J Hematol. 2015;90(3):220-4. DOI 10.1002/ajh.23911.

4. Morimoto A, Nakazawa Y, Ishii E. Hemophagocytic lymphohistiocytosis: Pathogenesis, diagnosis, and management. Pediatr Int. 2016;58(9):817-25. DOI 10.1111/ped.13064.

5. Esteban YM, de Jong JLO, Tesher MS. An Overview of Hemophagocytic Lymphohistiocytosis. Pediatr Ann. 2017;46(8):e309-e13. DOI 10.3928/1938235920170717-01.

6. Larroche C. Hemophagocytic lymphohistiocytosis in adults: diagnosis and treatment. Joint Bone Spine. 2012;79(4):356-61. DOI 10.1016/j.jbspin.2011.10.015.

7. Wysocki CA. Comparing hemophagocytic lymphohistiocytosis in pediatric and adult patients. Curr Opin Allergy Clin Immunol. 2017;17(6):405-13. DOI 10.1097/ACI.0000000000000405.

8. Nieto JF, Gomez SM, Moncada DC, Serna LM, Hidron AI. Successful treatment of hemophagocytic lymphohistiocytosis and disseminated intravascular coagulation secondary to histoplasmosis in a patient with HIV/AIDS. Biomedica. 2016;36(0):9-14. DOI 10.7705/ biomedica.v36i2.2797.

9. Nieto-Ríos JF, Serna-Higuita LM, Guzman-Luna CE, Ocampo-Kohn C, Aristizabal-Alzate A, Ramírez I, et al. Histoplasmosis in renal transplant patients in an endemic area at a reference hospital in Medellin, Colombia. Transplant Proc. 2014;46(9):3004-9. DOI 10.1016/j.transproceed.2014.06.060.

10. Nieto-Ríos JF, Aristizabal-Alzate A, Ocampo C, Serrano-Gayubo AK, Serna-Higuita LM, Zuluaga-Valencia G. Disseminated histoplasmosis and haemophagocytic syndrome in two kidney transplant patients. Nefrologia. 2012;32(5):683-4. DOI 10.3265/Nefrologia. pre2012.Jun. 11508 .

11. Filippone EJ, Singh P, Frank AM, Gupta A, Farber JL. Rapidly Fatal Hemophagocytic Lymphohistiocytosis Developing Within Six Days Following Deceased-Donor Renal Transplantation: Case Report. Transplant
Proc. 2016;48(9):3123-7. DOI 10.1016/j.transproceed.2016.03.031.

12. Yaich S, Charfeddine K, Hsairi D, Zaghdane S, Kammoun K, Makni S, et al. BK virus-associated hemophagocytic syndrome in a renal transplant recipient. Saudi J Kidney Dis Transpl. 2014;25(3):610-4.

13. Lehmberg K, Nichols KE, Henter JI, Girschikofsky M, Greenwood T, Jordan M, et al. Consensus recommendations for the diagnosis and management of hemophagocytic lymphohistiocytosis associated with malignancies. Haematologica. 2015;100(8):997-1004. DOI 10.3324/haematol.2015.123562.

14. Vega J, Rodriguez M, Goecke H, Santamaria H. Síndrome hemofagocítico en un trasplantado renal con síndrome de Alport. Rev Med Chile. 2013;141:519-24. DOI 10.4067/S0034-98872013000400014.

15. Karras A, Thervet E, Legendre C, Groupe Cooperatif de transplantation d'Ile de F. Hemophagocytic syndrome in renal transplant recipients: report of 17 cases and review of literature. Transplantation. 2004;77(2):23843. DOI 10.1097/01.TP.0000107285.86939.37.

16. Marinella MA. Hematologic abnormalities foIlowing renal transplantation. Int Urol Nephrol. 2010;42(1):151-64. DOI 10.1007/s1 1255-009-9558-5.

17. Nusshag C, Morath C, Zeier M, Weigand MA, Merle U, Brenner T. Hemophagocytic lymphohistiocytosis in an adult kidney transplant recipient successfully treated by plasmapheresis: A case report and review of the literature. Medicine (Baltimore). 2017;96(50):e9283. DOI 0.1097/MD.0000000000009283.

18. Segall L, Moal MC, Doucet L, Kerooat N, Bourbigot B. Toxoplasmosis-associated hemophagocytic syndrome in renal transplantation. Transpl Int. 2006;19(1):7880. DOI 10.1111/j.1432-2277.2005.00179.x.

19. Ardalan MR, Shoja MM, Tubbs RS, Esmaili H, Keyvani H. Postrenal transplant hemophagocytic lymphohistiocytosis and thrombotic microangiopathy associated with parvovirus b19 infection. Am J Transplant. 2008;8(6):1340-4. DOI 10.1111/j.16006143.2008.02244.x.

20. Lo MM, Mo JQ, Dixon BP, Czech KA. Disseminated histoplasmosis associated with hemophagocytic lymphohistiocytosis in kidney transplant recipients. Am J Transplant. 2010;10(3):687-91. DOI 10.1111/j.16006143.2009.02969.x.

21. Ponticelli C, Alberighi OD. Haemophagocytic syndrome--a life-threatening complication of 
renal transplantation. Nephrol Dial Transplant. 2009;24(9):2623-7. DOI 10.1093/ndt/gfp282.

22. Daver N, McClain K, Allen CE, Parikh SA, Otrock Z, Rojas-Hernandez $C$, et al. A consensus review on maliognancy-associated hemophagocytic lymphohistiocytosis in adults. Cancer. 2017;123(17):3229-40. DOI 10.1002/cncr.30826.

23. Nieto-Rios JF, Gómez de Los Ríos SM, Serna-Higuita LM, Ocampo-Kohn C, Aristizabal-Alzate A, GálvezCárdenas KM, et aI. Treatment of post-transplantation lymphoproliferative disorders after kidney transplant with rituximab and conversion to m-TOR inhibitor. Colomb Med. 2016;47(4):196-202.

24. Nieto-Rios JF, Gómez de Los Ríos SM, Serna-Higuita LM, Ocampo-Kohn C, Aristizabal-Alzate A, Gálvez-Cárdenas KM, et al. Treatment of post-transplantation lymphoproliferative disorders after kidney transplant with rituximab and conversion to $\mathrm{m}-\mathrm{TOR}$ inhibitor. Colomb Med. 2016;47(4):196-202.

25. Nieto-Ríos JF, Gómez de Los Ríos SM, Serna-Higuita LM, Gálvez-Cardenas KM. Enfermedad linfoproliferativa postrasplante de órogano sólido. Iatreia. 2016;29(3):312-22.

26. Hust MA, Blechacz BRA, Bonilla DL, Daver N, RojasHernandez CM. Adult cancer-related hemophagocytic lymphohistiocytosis - a challenging diagnosis: a case report. J Med Case Rep. 2017;11(1):172. DOI 10.1186/s13256-017-1344-x.

27. Wakefield C, Mehta PA, Corathers S, Geller J, Gelfand M, Olowokure O, et al. A First Report of Secondary Hemophagocytic Lymphohistiocytosis Associated With Papillary Thyroid Carcinoma. J Pediatr Hematol Oncol. 2018;40(2):e97-e8. DOI 10.1097/ MPH.0000000000000991.

28. Satzoger I, Ivanyi P, Langer F, Kreipe HH, SchaperGerhardt K, Beutel G, et al. Treatment-related hemophagocytic lymphohistiocytosis secondary to checkpoint inhibition with nivolumab plus ipilimumab. Eur J Cancer. 2018;93:150-3. DOI 10.1016/j. ejca.2018.01.063.

29. Janka GE, Lehmbero K. Hemophagocytic syndromes--an update. Blood Rev. 2014;28(4):135-42. DOI 10.1016/j.blre.2014.03.002.

30. Voskoboinik I, Smyth MJ, Trapani JA. Perforin-mediated target-cell death and immune homeostasis. Nat Rev Immunol. 2006;6(12):940-52. DOI 10.1038/nri1983.
31. Filippone EJ, Farber JL. Hemophagocytic lymphohistiocytosis: an update for nephrologists. Int Urol Nephrol. 2016;48(8):1291-304. DOI 10.1007/s11255-0161294-z.

32. Weaver LK, Behrens EM. Hyperinflammation, rather than hemophagocytosis, is the common link between macrophage activation syndrome and hemophagocytic lymphohistiocytosis. Curr Opin Rheumatol. 2014;26(5):562-9. DOI 10.1097/ BOR. 0000000000000093 .

33. Weaver LK, Behrens EM. Weathering the storm: Improving therapeutic interventions for cytokine storm syndromes by targeting disease pathogenesis. Curr Treatm Opt Rheumatol. 2017;3(1):33-48. DOI 10.1007/ s40674-017-0059-X.

34. Canna SW, Behrens EM. Making sense of the cytokine storm: a conceptual framework for understanding, diagnosing, and treating hemophagocytic syndromes. Pediatr Clin North Am. 2012;59(2):329-44. DOI 10.1016/j.pcl.2012.03.002.

35. De Saint Basile G, Menasche G, Fischer A. Molecular mechanisms of biogenesis and exocytosis of cytotoxic granules. Nat Rev Immunol. 2010;10(8):568-79. DOI $10.1038 /$ nri2803.

36. Thiery J, Keefe D, Boulant S, Boucrot E, WaIch M, Martinvalet D, et al. Perforin pores in the endosomal membrane trigger the release of endocytosed granzyme B into the cytosol of target cells. Nat Immunol. 2011;12(8):770-7. DOI 10.1038/ni.2050.

37. Marsh RA. Epstein-Barr Virus and Hemophagocytic Lymphohistiocytosis. Front Immunol. 2017;8:1902. DOI 10.3389/fimmu.2017.01902.

38. Arlet JB, Le TH, Marinho A, Amoura Z, Wechsler B, Papo T, et al. Reactive haemophagocytic syndrome in adult-onset Still's disease: a report of six patients and a review of the literature. Ann Rheum Dis. 2006;65(12):1596-601. DOI 10.1136/ard.2005.046904.

39. Esmaili H, Mostafidi E, Mehramuz B, Ardalan M, Mohajel-Shoja M. An update on renal involvement in hemophagocytic syndrome (macrophage activation syndrome). J Nephropathol. 2016;5(1):8-14. DOI 10.15171/jnp.2016.02.

40. Thaunat O, Delahousse M, Fakhouri F, Martinez F, Stephan JL, Noel LH, et al. Nephrotic syndrome associated with hemophagocytic syndrome. Kidney Int. 2006;69(10):1892-8. DOI 10.1038/sj.ki.5000352. 
41. AI-Lamki RS, Mayadas TN. TNF receptors: signaling pathways and contribution to renal dysfunction. Kidney Int. 2015;87(2):281-96. DOI 10.1038/sj.ki.5000352.

42. Allen CE, Yu X, Kozinetz CA, McClain KL. Highly elevated ferritin levels and the diagnosis of hemophagocytic lymphohistiocytosis. Pediatr Blood Cancer. 2008;50(6):1227-35. DOI 10.1002/pbc.21423.

43. Lehmberg K, McClain KL, Janka GE, Allen CE. Determination of an appropriate cut-off value for ferritin in the diagnosis of hemophagocytic lymphohistiocytosis. Pediatr Blood Cancer. 2014;61(11):2101-3. DOI 10.1002/pbc.25058.

44. Otrock ZK, Daver N, Kantarjian HM, Eby CS. Diagnostic Challenges of Hemophagocytic Lymphohistiocytosis. Clin Lymphoma Myeloma Leuk. 2017;17S:S105-S10. DOI 10.1016/j.clmI.2017.02.017.

45. George MR. Hemophagocytic Iymphohistiocytosis: review of etiologies and management. J Blood Med. 2014;5:69-86. DOI 10.2147/JBM.S46255.

46. Henter JI, Horne A, Arico M, Egeler RM, Filipovich AH, Imashuku S, et al. HLH-2004: Diagnostic and therapeutic guidelines for hemophagocytic lymphohistiocytosis. Pediatr Blood Cancer. 2007;48(2):124-31. DOI 10.1002/pbc.21039.

47. Asci G, Toz H, Ozkahya M, Cagirgan S, Duman S, Sezis $M$, et al. High-dose immunoglobulin therapy in renal transplant recipients with hemophagocytic histiocytic syndrome. J Nephrol. 2006;19(3):322-6.

48. Caselli D, Arico M, Party EPW. The role of BMT in childhood histiocytoses. Bone Marrow Transplant. 2008;41 Suppl 2:S8-S13. DOI 10.1038/bmt.2008.46.

49. Lin S, Li Y, Long J, Liu Q, Yang F, He Y. Acute liver failure caused by hemophagocytic lymphohistiocytosis in adults: A case report and review of the literature. Medicine (Baltimore). 2016;95(47):e5431. DOI 10.1097/MD.0000000000005431.

50. Pastula DM, Burish M, Reis GF, Bollen A, Cha S, Ralph J, et al. Adult-onset central nervous system hemophagocytic lymphohistiocytosis: a case report. BMC Neurol. 2015;15:203. DOI 10.1186/s12883-015-0470-6

51. Machaczka M, Vaktnas J, Chiang SC, Bryceson YT. Alemtuzumab treatment for hemophagocytic lymphohistiocytosis. Nat Rev Clin Oncol. 2010;7(10). DOI 10.1038/nrclinonc.2010.40-c1. 